\section{PSYCHOSTATS: BASIC programs for data analysis in psychology}

\author{
LESTER H. GILBERT \\ University of Cape Town \\ Rondebosch 7700, Cape, South Africa
}

The PSYCHOSTATS package provides a well stocked toolbox for the psychologist intent upon the analysis of experimental data. Eight sections (21 programs) are devoted to the analysis of variance, three sections (6 programs) to the analysis of multivariate data, and two sections ( 9 programs) to nonparametric techniques. The list of programs is as follows: (1) student's $t$ testssingle, independent, and dependent samples; (2) one-way ANOVAs-simple, with repeated measures; (3) two-way ANOVAs-simple, with repeated measures on Factor B, and with repeated measures on Factors $\mathbf{A}$ and $\mathbf{B}$; (4) three-way ANOVAs-simple, with repeated measures on Factor $\mathrm{C}$, and with repeated measures on Factors $\mathrm{B}$ and $C$; (5) multiple and pairwise comparisons-one-way, two-way, and three-way ANOVAs; (6) simple main effects-two-way and three-way ANOVAs; (7) Latin squares-simple and with repeated measures on Factors B and C; (8) analyses of covariance-one-way, one-way with repeated measures, and two-way; (9) Hotelling's T-squared-independent and dependent samples; (10) correlation-simple matrix and with missing values; (11) regression-simple linear and stepwise multiple; (12) nonparametric-Mann-Whitney, Wilcoxon, Spearman, Kendall, Kruskal-Wallis, Friedman, and Cochran; and (13) chi square-single and double classification. In addition, two FORTRAN programs are provided for factor analysis, for teaching rather than analytic purposes.

\section{Design Philosophy}

The package is coherently designed with certain criteria. It is meant to provide a set of easily used statistical routines for users with access to a minicomputer or other small computer system. No mass storage is required. The BASIC programming language is used, since it is easily learned and the programs are easily modified. Minimum core storage is assumed: The system is at present running on a Hewlett-Packard 2114B 16-bit $8 \mathrm{~K}$ minicomputer, with about $2 \mathrm{~K}$ available to the user after the BASIC interpreter is loaded. Almost any machine with $4 \mathrm{~K}$ bytes available to the user will accept all PSYCHOSTATS programs. To achieve this, the more complex programs are constructed as overlays.

Extensive Documentation. The primary feature of the package is the documentation. For each program, an abstract description is provided, with limitations, method of use, mathematical method, worked example, complete listing, and notes and references where applicable. Every attempt has been made to keep the terminology consistent from program to program; the terminology has been taken from Kirk (1968) and Winer (1971). As much as possible, the programs are self-explanatory when running, so as to minimize reference to the documentation.

Interactive. For the user, the primary feature of the programs is that they are interactive. Parameter values are requested with questions such as "NO OF LEVELS OF FACTOR A?", rather than with obscure mnemonics. Furthermore, all parameter requests occur at the start of a program, since errors in parameter specifications are irrecoverable.

Error Correction. Errors in raw data entry are recoverable in all programs. Such a feature is essential where thousands of data items could potentially be input.

Thoroughness. Most computer packages for data analysis only provide for the first level of analysis and leave the user to complete any follow-up computations that may be necessary. Typically, significant results in the analysis of variance require further analysis of simple main effects or the examination of pairwise and multiple contrasts. PSYCHOSTATS provides programs to carry out these second-level analyses using summary data from the first level.

Other Features. The ANOVA programs provide checks on the assumption of homogeneity of variance. Unequal sample sizes are allowed through an unweighted means analysis, means and standard deviations are provided liberally, and raw data need only be entered once, such that following programs accept summary data for further processing.

\section{Availability}

The package is available in the form of a system manual, an operator's manual, and a programmer's manual from the author, Department of Psychology, University of Cape Town, Private Bag, Rondebosch 7700, Cape, Republic of South Africa. No charge is made other than for printing and postage costs, which are about $\$ 13$. Duplication of any manual or part is allowed, provided the copies are retained by the purchasing institution.

\section{REFERENCES}

KIRK, R. E. Experimental design: Procedures for the behavioral sciences. Belmont, Calif: Brooks/Cole, 1968.

Winer, B. J. Statistical principles in experimental design (2nd ed.). New York: McGraw-Hill, 1971.

(Accepted for publication June 8, 1979.) 